\title{
Sobre el análisis y representación de documentos
}

\author{
RAMIRO LAFUENTE LÓPEZ \\ Centro Universitario de Investigaciones Bibliotecológicas \\ de la UNAM, 04510, México, D.F., Tel: (525)623-03-29 \\ E-mail: alaf@axtel.net
}

\begin{abstract}
RESUMEN
El autor presenta en este artículo una primera aproximación a los problemas relativos a la comprensión de lo que significa el análisis y representación de documentos.

Palabras clave: Análisis Documental, Descripción Bibliográfica, Normalización.
\end{abstract}

\section{ON THE ANALYSIS AND REPRESENTATION OF DOCUMENTS RAMIRO LAFUENTE-LÓPEZ}

Trabajo recibido el 7 de julio de 2001

*

Trabajo aceptado el 15 de julio de 2001

\begin{abstract}
ABst RACT
The author presents in this article a first approach to the problems relating to the understanding of what is meant by the analysis and representation of documents.

Key words: Document Analysis, Bibliographic Description, Standardization.
\end{abstract}

\section{DEL ANÁLISIS DE DOCUMENTOS}

I 1 término análisis documental alude al conjunto de conocimientos relativos a $ـ$ los principios, métodos y técnicas que permiten examinar, distinguir y separar cada una de las partes de un documento, para determinar la categoría a que pertenece, su estructura formal, propiedades y significado de sus contenidos temáticos. Se trata, pues, de un método de conocimiento que facilita el estudio de los documentos ya sea en grupo 0 aisladamente.

Los resultados del análisis de grupos de documentos se expresan en forma de categońas dedoumentos es decir, en conceptos abstractos que definen sus propiedades comunes y sus relaciones más generales. Estas categorías son el resultado de una abstracción que generaliza los aspectos particulares o singulares de los documentos que produce y utiliza una comunidad.

Las categorías son un elemento para el estudio y clasificación, y pueden referirse a los modos de organización de la producción y uso de documentos que generalmente se expresan en forma de tipologías. Asimismo pueden crearse categorías que eluciden las propiedades comunes y las relaciones más generales de la estructura formal de los documentos. Normalmente esto se logra a través de sistemas de 
reglas para describirlos, de manuales de estilo para producirlos y publicarlos, o de sistemas de normas para regular su apariencia.

Las formas para la creación de documentos ha sido motivo de estudio de diversas disciplinas y ha generado una amplia literatura relativa a los conocimientos necesarios para construir y validar un determinado tipo de documentos.

En la sociedad contemporánea la difusión, publicación y uso de documentos es de tal magnitud que ha dado lugar a la creación de documentos denominados seandanios los cuales describen sistemáticamente a otros documentos denominados, obviamente, primarios

La descripción de los documentos se hace mediante la expresión abreviada de sus características formales y del significado de sus contenidos para facilitar la formación de acervos y el acceso a los mismos. Las descripciones de forma y contenido de un documento intentan representarlo en forma unívoca y singular, tanto para distinguirlo individualmente como para expresar sus relaciones generales dentro de la producción documental, y con ello crear medios para su localización y adquisición. D e hecho se da por sentado que ante la ingente producción de diversos tipos de documentos, es prácticamente imposible que una sola persona pueda tener un acceso directo y simple a todos ellos. El sujeto difícilmente puede tener noticia de todo lo que circula y se enfrenta al problema de ubicar un documento en particular. ${ }^{1}$

La creación y construcción de doumentos seundanios, como índices, catálogos y bibliografías, se realiza básicamente con fines de orientación científica e informativa para representar, sintéticamente, tanto el continente como el contenido de los documentos originales.

Se presupone que los documentos secundarios, al contener información concentrada y sistematizada, ofrecen cierta facilidad para obtener referencias de los materiales originales o primarios. Los documentos secundarios, pues, pueden contener la producción general o parcial de documentos, como es el caso de los catálogos editoriales y bibliográficos, o bien, pueden referir la existencia de colecciones documentales administradas por instituciones, como es el caso de los catálogos de bibliotecas.

El diseño, construcción y operación de bibliotecas está sustentado en la selección, formación y administración de colecciones de documentos, pero también en el diseño y construcción de documentos secundarios, como índices, catálogos y bibliografías, con el objetivo de facilitar la ubicación y localización de materiales dentro de los acervos de las instituciones - dedicadas a formarlos para utilizarlos en forma colectiva- 0 en el mercado editorial.

1 Cfr. María Rosa Garrido Arilla. Teońa ehistaria dela catalogacón de doumentos Madrid : Editorial Síntesis, 1996, pp. 17-24. 
Los conocimientos sobre estos procesos están referidos básicamente a los impresos, no obstante, con la automatización y digitalización de publicaciones ha cambiado la estructura formal de los documentos secundarios, toda vez que se tiende de manera creciente a construirlos como documentos en línea para su uso vía intranets o Internet.

La difusión de la producción editorial se sustenta en la idea de autores propietarios de impresos, que si bien son independientes entre sí, constituyen un monopolio; sin embargo no están aislados unos de otros, puesto que el significado de los contenidos temáticos de los impresos que generan mantienen un vínculo en razón de una serie de creencias - sustento del desarrollo de la actividad científica, académica, de divulgación, difusión o entretenimiento- que facilitan el que las publicaciones producidas adquieran sentido y significado para distintas comunidades. Así por ejemplo, la actividad científica considera que la publicación debe servir al propósito de recrear y acumular el conocimiento, de manera que un autor retoma partes de otro autor y los incorpora a su propia publicación y así sucesivamente.

La actividad académica establece que el aprendizaje debe apoyarse en el conocimiento de los significados de esas publicaciones y crea bibliotecas como un instrumento para construir acervos clasificados de uso colectivo que faciliten su acceso.

El análisis documental se desarrolla con la finalidad de crear métodos y técnicas para analizar documentos, clasificar acervos y con ello facilitar su uso colectivo. Ello se fundamenta en la idea de que los significados de los contenidos temáticos de alguna manera se ligan con los significados de otros, lo cual permite crear esquemas de clasificación capaces de abarcar los significados comunes a varios tipos de documentos. Esta idea de representación de documentos, independientes unos de otros, posibilita particularizar su contenido temático y establecer los elementos necesarios para vincularlos con los contenidos de otros documentos.

La creciente presencia de la automatización obligó a revisar las formas y estructuras de los documentos secundarios convencionales, de los que incluso se generaron versiones en línea. Sin embargo, la innovación tecnológica que representa la automatización hace inevitable reconsiderar los conceptos acerca del análisis y representación de documentos, sobre todo porque hasta ahora únicamente se ha estudiado la estructura de los impresos y no existen acuerdos o convenciones generalizadas acerca de los documentos digitales, aunque es previsible que la tendencia en la estructuración de éstos, sustentada en el uso de lenguajes de marcado como el SGML y sus derivados, sea la tendencia que prevalezca.

Los sistemas para almacenar información cuentan con elementos que permiten 1) almacenar los documentos que se adquieren; 2) organizar y controlar los documentos que se adquieren en función de las diferentes demandas de los usuarios (catalogación, indización, clasificación) de modo que puedan ser identificados fácilmente; 3) describir físicamente el documento para analizar su contenido conceptual y traducirlo a un vocabulario determinado - que constituya una representación 
del mismo- para finalmente almacenarlo en una base de datos que sirva como instrumento de salida del sistema. ${ }^{2}$

\section{Ámbito del análisis documental}

El ámbito del análisis documental comprende el estudio de los principios, conceptos, técnicas y métodos que permiten formular enunciados cuya función es expresar una idea acerca de un documento por medio de palabras, signos y códigos convencionales, con la intención de que éstos constituyan una representación que haga las veces del documento a fin de poder identificarlo, clasificarlo y localizarlo.

La representación de un documento a través de símbolos está condicionada por el sujeto que la realiza, es decir por los objetivos que persigue, así como por las formas generales de la representación que pertenecen al sujeto y no a los elementos del documento. ${ }^{3}$

\section{Representar documentos}

Al representar un documento se construye una imagen o un símbolo del mismo y convencionalmente se acepta que éste permite saber acerca del material original. Lo cual no implica que los enunciados que se edifican lleven a conocer el documento, simplemente son formulaciones que facilitan el saber acerca del mismo.

Conocer un documento implica un nivel de mayor profundidad, a saber: leerlo, comprenderlo, ubicar sus contenidos temáticos como parte de otros contenidos temáticos; supone, además, integrar todos los elementos acerca de un documento en una sola unidad. La condición para conocer un documento es captarlo en sus diferentes matices, bajo diferentes perspectivas y, eventualmente, en situaciones distintas. Supone también tener experiencias variadas sobre el mismo y poder hacer, de algún modo, una serie de inferencias a partir de ellas, referidas al documento en cuestión.

Cuando nuestro conocimiento es circunstancial y hablamos de "conocer" conforme a su significado semántico común, sólo podemos referirnos a aspectos superficiales y aun ocasionales del documento. Sin embargo, conocer un documento implica poder contestar múltiples cuestiones de diverso índole. Pensemos en el siguiente enunciado: "Conoce las obras de Villoro". En esta frase se supone

2 Cfr. Frederick W. Lancaster. El control dd vocabulario en la reauperación de informadón 2a. ed., Valencia, España : Universitat de València, 1995, 286 p.

3 Partimos de la idea del documento como un todo integrado. El distinguir forma y contenido únicamente tiene sentido para fines metodológicos de análisis. Algunos autores conciben el documento como poseedor de una doble naturaleza: soporte más contenido, es decir, el documento estaría integrado por dos elementos dicotómicos, la información en él contenida y su soporte documental. Un resumen sobre estas ideas puede consultarse en: Adelina Clauso G arcía. "Análisis documental: el análisis formal". Revista Geneal de Infomadón y Doumetaaión vol. 3(1), 11-19, 1993. 
que quien conoce puede ser una fuente de información variada sobre los libros, artículos, ponencias, etc., escritos por Villoro, y por tanto ser capaz de resolver problemas que le sean presentados respecto y orientar a otros. Porque conocer la obra de Villoro, en este sentido, no es sólo saber su descripción externa, sino captar su forma y manera, su estilo, el modo como sus partes están relacionadas en un todo, y ser capaz de relacionar entre sí las partes de su doctrina. Conocer un documento en su sentido más rico es poder integrar en una unidad cualquier experiencia y cualquier saber parcial sobre un documento, por variados que estos sean.

El que sabe muchas cosas acerca de un libro como El Quijotetiene con él una relación cognoscitiva diferente a quien realmente lo conoce. Q uien sabe del Quijote podrá citar frases del texto, dar noticias de sus diferentes ediciones y características de las mismas, incluso distinguir tipografía, papel y cambios en el texto, organización de los capítulos e ilustraciones que aparecen en distintas ediciones, y saber de las opiniones de distintas personas sobre el mismo. No obstante, quien conoce $\mathrm{El}$ Quijotetal vez no sepa nada de lo anterior, en cambio puede comprender su mensaje central, captar su espíritu, interpretar diversos asuntos a partir del mismo, saber responder preguntas acerca del alcance y aplicación de lo expuesto. Conocer El Quijoteno es saber muchas cosas acerca de él, sino poder distinguir lo esencial de sus contenidos temáticos y literarios, "núcleo" del que puede desprenderse cualquier formulación parcial.

Conocer un documento implica integrar lo que se sabe del mismo a modo de comprender lo central de sus contenidos y captar su articulación interna. Es distinto "saber sobre el libro El Juegodela lóġa escrito por Lewis Carroll" a "conocer el libro El Juegodela lóġa escrito por Lewis Carroll". Lo primero es poder describir lo que se sabe, o bien, exponerlo parte por parte; lo segundo es haberlo comprendido en su estructura y poder, en consecuencia, distinguir en él lo relevante y exponerlo como un conjunto coherente.

Conocer no es una suma de saberes, sino una fuente de ellos, implica tener un modo de relacionar cualquier saber específico con otros saberes. El saber, en cambio, es necesariamente parcial, mientras que el conocer aspira a captar una totalidad. D istinguir entre saber y conocer ${ }^{4}$ permite establecer diferentes niveles o modos de representación de un documento, e implica distintas modalidades de aproximación al mismo. El saber proporciona una interpretación descriptiva del documento; el conocer, en cambio, conduce a su comprensión.

La representación de un documento se expresa de diversas formas, por ejemplo una ficha catalográfica, un resumen, una reseña, una ficha bibliográfica, una referencia, palabras clave, entre otras. Los enunciados y formulaciones que se realizan para nombrar un documento como un todo, constituyen una representación del mismo.

4 Crr. Villoro. Saber y conoer. México, Siglo Xxi, p. 197 y ss. 
Una colección, agrupamiento, agregado o lista de representaciones de documentos conforman un universo cuya definición depende del contexto en que se utiliza o del problema que se trata de resolver. Existen diversos tipos de ellos, como pueden ser: catálogos, índices, bibliografías, bases de datos bibliográficos, entre otros.

Para facilitar la representación de un documento se definen conceptos y procedimientos en forma de sistemas de reglas que establecen conceptos, principios generales, procesos y técnicas para representar a un documento con la intención de incluirlo como parte de un universo siempre en proceso de construcción, y que podrá estar constituido por un número indeterminado de representaciones diversas.

Las reglas se pueden organizar:

* conforme al tipo de representación, en cuyo caso las reglas se orientan a la construcción de algún tipo de representación, como puede ser la simple descripción física de un documento, o bien, tratarse de formas más complejas como es el caso de los resúmenes o las reseñas.

* conforme a las caractenísticas de una clase de documentos, en cuyo caso se agrupan y jerarquizan los elementos que se consideran peculiares para representarlos dentro de una clase específica.

La representación de un documento no debe contemplarse como un hecho aislado, al contrario siempre debe concebirse como parte de otras representaciones, con objeto de facilitar su agrupación de distintas maneras, para posibilitar la creación de diversas clases de colecciones de representaciones, como puede ser un catálogo de biblioteca, una bibliografía, una base de datos, entre otras. Cada una de las cuales cumple diversas finalidades y define las características de la colección en cuestión.

La representación de los documentos y sus contenidos debe estar orientada a patentizar frente al público tanto las características físicas y/ o contenidos temáticos como las relaciones que guardan entre sí los diferentes documentos que forman una colección. La representación de documentos puede expresarse de dos formas:

a) por medio de símbolos que representan los contenidos documentales y sirven para acomodar los documentos en un orden predeterminado, y/ o

b) a través de un registro que contiene la descripción de las características físicas y/ o temáticas.

Tanto los símbolos creados a partir de un sistema de clasificación (D ewey, LC, $\mathrm{CDU}$, u otros) como los registros realizados en forma de fichas o registros electrónicos utilizados para representar un documento (libro, material hemerográfico, revista, vídeo, etc.), muestran las relaciones entre diferentes fenómenos vinculados a la producción y uso de documentos (pueden referirse a sus características físicas y/ o a sus contenidos temáticos). 
Al representar algún tipo de documento por medio de un registro, construido específicamente para figurar en lugar de éste, se pretende que tenga las cualidades necesarias para relacionar cualquiera de los elementos incluidos como parte de un registro con los elementos de otro. Estas relaciones tienen la finalidad de crear un orden que explicite los vínculos.

Por ejemplo, cuando decimos "el libro escrito por G arcía Márquez se titula Cien añosdeSdeedad", estamos expresando una relación entre un sujeto: "un libro escrito por García Márquez", y un objeto en particular "el libro titulado Cien años desde dad". Además, esta relación tiene un orden específico: es el libro escrito por G arcía Márquez el que tiene el título Cienañosdesdecad, y no es el título el que tiene un libro.

Algunas relaciones que aparecen en los registros no siempre mencionan todos los elementos que entran en juego. Así, cuando decimos "el libro titulado El Hom breGramatical es un libro de divulgadón dentífica", queremos decir que existe una categoría, denominada divlgacóncentífica, que se aplica al libro El HombreGramatical. Sin embargo, no se señala quién considera que esta obra es de divulgación científica ni por qué. Así pues, detallar todos los fenómenos implicados en una relación depende de qué es lo que se quiere decir, y de la finalidad que se persigue al construir una representación.

Existen diversos modos, métodos y técnicas para construir representaciones, pues la invención humana no sólo modifica la selección y agrupación de los elementos que las constituyen, sino que la conformación misma puede organizarse so bre la base de la homologación de distintas formas, en atención al cumplimiento de propósitos diversos relacionados con la organización de colecciones documentales y con la creación y operación de redes de información.

En este sentido es factible retomar conceptos y principios de la lógica, así como las técnicas establecidas en diversos sistemas de reglas y normas - siempre y cuando se eviten posibles contradicciones- y emplearlos para construir políticas, lineamientos o sistemas normativos específicos. Sería el caso, por ejemplo, de los estilos bibliográficos que utilizan diferentes revistas científicas, donde cada uno de ellos obedece a una lógica y al cumplimiento de finalidades específicas.

Para evitar que un sistema normativo quedase aislado es necesario homologarlo con otros, es decir hacer sus reglas y resultados equiparables a las de otros sistemas semejantes. El conocimiento compartido sobre los modos de representación documental permite cifrar y descifrar mensajes entre distintos tipos de interlocutores. Estos modos son producto de conocimientos acerca de la naturaleza de distintas clases de documentos que se expresan en forma de sistemas de reglas cuyos enunciados permiten producir e interpretar sus representaciones.

Los principios generales y las reglas a que nos referimos sirven al propósito de generar sistemas de significación que permitan organizar las representaciones en clases o tipos. Sin embargo no es factible la existencia de un solo tipo, forma 0 modo para representar documentos, toda vez que, en términos generales, cada 
comunidad tiene un conjunto de enunciados con los cuales, los practicantes de una disciplina, la definen, trazan sus bordes externos e internos y sus rutas interiores por medio del lenguaje y sus significados. Estos enunciados delimitan la función del sujeto (poeta, bibliotecario, ingeniero, médico, artista) y la definición de los principios generales de la actividad de la disciplina, estableciendo lo que le es pertinente, así como los conceptos y estructuras de discursos pertenecientes a la disciplina; en su caso, también determinan la metodología y los principios epistemológicos. Son enunciados en los que reconocemos una actividad disciplinaria y con ellos se genera una tipología útil para la representación documental.

Representar, pues, adquiere sentido únicamente en el contexto de necesidades y conductas de los miembros de una comunidad. El proceso intelectual de interpretación de la forma y/ o contenidos temáticos de los documentos - para representarlos por medio de códigos o palabras- únicamente adquiere sentido cuando está orientado al cumplimiento de intencionalidades o finalidades que se expresan a través de algún tipo de producto o servicio (formación de acervos, préstamo, servicio de información, o el clásico catálogo). Las finalidades dotan a la representación de significados relevantes para los miembros de una comunidad, al hacer patente el medio que el público tiene para beneficiarse de la organización de documentos que se logra por medio de su análisis y representación.

A hora bien, si nos atenemos al hecho de que a los servicios es necesario expresarlos por medio de sistemas de control administrativo, de instrumentos de acceso y de obtención de documentos, nos encontramos entonces frente a un asunto álgido. Si el proceso organizador de los servicios se orienta exclusivamente hacia la búsqueda de la eficiencia y margina la disponibilidad de medios para hacer explícitas las intenciones, significados y finalidades de la representación de documentos, indudablemente estaremos frente a la presencia de una maquinaria administrativa que puede ser impresionantemente eficaz para resolver los problemas estándar de almacenamiento y recuperación de información, pero que poco tendrá para ofrecer al individuo que busca información o conocimientos. Por ello es fundamental que exista coherencia, así como vínculos y relaciones entre los servicios, el análisis y la representación de documentos, situación que se rompió al dividir el trabajo y separar a uno de otro.

El análisis de documentos implica preguntarse acerca de la naturaleza del diálogo que se presenta o debe presentarse con la comunidad para la cual se realiza, asunto que evidentemente está relacionado con aspectos inherentes a la lógica de la construcción de sistemas de clasificación y a la determinación de significados y relevancia que se le otorguen a los contenidos de los documentos. Pero sobre todo, implica establecer las cualidades que deben reunir los servicios por medio de los cuales se pretende responder a las necesidades de un mundo que induce al uso de la información en forma rápida y precisa; y, al mismo tiempo, requiere de elementos y espacios para que el individuo pueda generar e integrar conocimientos 
que le permitan no sólo explicarse lo que sucede en el mundo, sino que contribuyan a enriquecer su concepción individual del mismo; a fin de cuentas la lectura de compresión en primer término le sirve para estructurar o reestructurar sus propias concepciones.

En las últimas décadas ha prevalecido la idea de sustentar el análisis y la representación de documentos en la concepción del almacenamiento y recuperación de la información, cuya intencionalidad esencial radica en la construcción de técnicas que sean funcionales para hacer eficiente el proceso de búsqueda, por medio de servicios orientados a proveer de información al usuario. La organización de colecciones de documentos así como los instrumentos de acceso se dirigen a la localización de información y datos específicos, relegando a un segundo plano el establecer y mostrar las relaciones que existen entre diferentes documentos. Al sustentar la búsqueda y recuperación de información en las ideas acerca de la relevancia y la pertinencia de ésta, se producen dos fenómenos: la sobreinformación, porque la eficacia de los sistemas de almacenamiento y recuperación produce más información de la que un individuo puede asimilar; o bien la desinformación, dado que al sujeto no le es fácil recuperar información pertinente. Esta situación se presenta porque no es fácil determinar la relevancia y la pertinencia de un documento, ya que éste adquiere esos valores en función de las necesidades específicas de información de un sujeto, y no por sí mismo.

Estos conceptos se introdujeron como parte de las técnicas de búsqueda de información. Fueron derivados de las concepciones de la escuela lingüística de Praga, y se han definido de la siguiente forma:

* Relevancia: califica los rasgos significativos y operaciones lógicas (exclusión, suma, pertenencia, etc.) que tienen un valor diferencial en la selección de términos para una búsqueda de información.

* Pertinencia: califica los rasgos significativos que tienen un valor diferencial en la selección de los datos referentes a la descripción de un documento, los cuales se obtienen por medio de una búsqueda de información y a la vez determinan el propósito de esta última.

Empero, consideramos que el aspecto fundamental que debe orientar el análisis para obtener representaciones de documentos no debe radicar exclusivamente en la idea de almacenar para recuperar, sino en una constante interrogación del texto que se trata de representar. La cuestión radica en encontrar los métodos y principios que permitan preguntarse acerca de las posibles relaciones entre los contenidos temáticos. Se trata de realizar procesos de inferencia que conduzcan a describir en forma de una secuencia los contenidos conceptuales del documento y sus relaciones con el contenido de otros, a fin de que las representaciones sirvan para que un sujeto pueda formarse un esquema de referencia acerca de los documentos analizados. 


\section{Sobre los modos para representar documentos}

El análisis y representación de un documento se apoya en un conjunto de principios, normas, técnicas y tecnologías que establecen lo que debe incluirse 0 no como parte de la descripción de un documento, o bien, indican los contenidos que debe tener para ser acreditado como perteneciente a un tema o considerarlo como parte de un tipo de documentos, o aun prescriben aquello que es necesario considerar para resumirlo o reseñarlo.

La construcción de categorías sistemáticas para establecer las condiciones o cualidades que deben considerarse para incluir un documento dentro de una clase perteneciente a una tipología, juega un papel destacado en la determinación de elementos que se utilizan para representarlo.

La tarea de la tipología de documentos, en el terreno de la representación de los mismos, no consiste en proponer definiciones ni en establecer clasificaciones ajenas al uso y características que diversas comunidades le asignan, sino en comprender los criterios empleados por ellas. A este respecto, y con referencia a la clasificación típica del libro en manuales, monografías, libros de texto, obras de consulta, etc., lo fundamental sería comprender porque se acepta y valida esta clasificación, además de que nos proporcionaría conocimientos para normar los criterios acerca de la representación.

Los criterios bajo los cuales deben clasificarse los documentos, para agruparlos en clases representativas - de los modos que tienen diferentes comunidades para registrar información y/ o conocimientos- , al margen de la naturaleza y calidad de su contenido, tienen su origen en:

* Conceptos inherentes al documento, por ejemplo las reflexiones de diferentes autores sobre la idea del valor del libro; las definiciones de la naturaleza del artículo científico (esto ha ocupado a diversos autores); o las opiniones sobre el documento digital.

* Reglas regulativas para su producción y uso, como es el caso de los manuales de estilo que establecen enunciados que describen las características formales que debe reunir determinado tipo de documento; los criterios de validación académica a tomar en cuenta para publicarlo; o las directrices editoriales de una revista.

* N ormas de carácter institucional o disciplinario, es el caso por ejemplo de los manuales técnicos como el marc, las reglas de catalogación, el ISBD , 0 los manuales de políticas de una biblioteca, entre otros. Cuya finalidad es establecer reglas de uso común que faciliten el control de acervos.

La tipología de documentos, como un modo de clasificación, adquiere distintos significados y formas conforme tiende a establecer sistemas de organización útiles para la representación de documentos cuyos contenidos se refieren a diferentes ramas del conocimiento, expresan distintos tipos de saberes, o el uso de la 
representación obedece a finalidades inherentes a una actividad específica, como puede ser la de identificar los documentos que se citan en un artículo científico.

Aquí, los sistemas de reglas, normas, técnicas y tecnologías abordan universos limitados, por ejemplo la descripción de monografías, o la forma de crear asientos para un catálogo. Aún cuando sistemas como las Reejaspara Catalogar, o las AACR2 pareciera que abordan un conjunto de principios muy amplio, su universo es tan limitado como puede ser la construcción de catálogos para bibliotecas. Estas reglas se organizan para solucionar problemas específicos. Fundamentalmente se responde a la pregunta ¿cómo puedo? que se expresa en forma de enunciados muy precisos: ¿cómo puedo registrar el asiento de un autor corporativo? ¿cuáles son los elementos para describir un video?

Para facilitar la enseñanza en esta materia algunos autores han establecido denominaciones para las diversas formas de agrupar conocimientos que sirvan a la representación de algunos tipos de documentos con fines específicos; sería el caso por ejemplo el caso de la catalogadón descriptiva, que alude a los conocimientos para describir documentos e incluir su representación como fichas de un catálogo.

Como parte del análisis de documentos podemos identificar tres niveles o clases de principios para:

* describir

* clasificar

* evaluar

La aplicación de estos principios produce como resultado distintos tipos de representaciones que pueden emplearse en forma combinada. Así por ejemplo, una reseña necesariamente incluye tanto la descripción del documento, como los elementos para clasificarlo e incluirlo como parte de un tema o corriente de pensamiento, e incluso los juicios acerca del valor del mismo.

\section{Representación documental y comunicación}

El estudio de las finalidades de la representación documental involucra problemas de significados en cuanto al uso que se le pretenda asignar a la producción de esas representaciones.

Un acervo de documentos es un acervo de conocimientos teóricamente disponibles para todos los hombres comunes, expertos o los bien informados, pues es la acumulación de la experiencia práctica, o la ciencia y la tecnología como concepciones fundamentales. Pero este acervo no está integrado, consiste en una mera yuxtaposición de sistemas de conocimiento que en sí no son coherentes, ni siquiera compatibles unos con otros y que para poder describirlos e integrarlos como una colección, es necesario formular representaciones que interpreten el significado de los diversos contenidos temáticos para poderlos relacionar o yuxtaponer como parte de un esquema de clasificación documental. 
Podríamos pensar en terrenos de significación en los niveles de comunicación entre el emisor y el receptor de información, lo cual plantearía una visión clara y evitaría caer en un laberinto del que no sería fácil salir porque nos permitiría saber y no precisamente creer lo que se informa.

En el primer terreno, el sujeto que informa y el que se informa tiene una significatividad primaria con una estructura clara y nítida, pues representa el mundo que está a nuestro alcance y que podemos observar de modo inmediato, y también, al menos en parte, dominar, o sea cambiar y reordenar mediante nuestras acciones. Es la zona dentro de la cual nuestros proyectos pueden ser materializados y concretados. El sujeto bien informado tiene claramente estructurado en su pensamiento el conocimiento de lo que puede transmitir.

En el segundo terreno de significación se plantea a nivel de pensamiento la existencia de campos no abiertos a nuestro dominio, pero vinculados de modo mediato al terreno de significatividad primaria, porque brindan las herramientas ya creadas que deben emplearse para alcanzar el fin proyectado, o porque establecen condiciones de las cuales depende la planificación misma o su ejecución. Basta con estar familiarizados con el objeto de conocer las posibilidades, probabilidades y riesgos que pueden contener con referencia a nuestro interés principal. D e esta manera, el sujeto bien informado y el hombre que se informa implican las relaciones de significado de forma autónoma e inmediata para conducirlo a una sola significación.

El siguiente terreno de significación podemos decir que por el momento no tiene vinculación con el significado de la información final; es relativamente no significativo, porque podemos seguir presuponiéndolo mientras no tenga lugar dentro de la información que pretendemos saber, ésta puede influir en los terrenos de significación que se manifiestan por medio de nuevas e inesperadas probabilidades o riesgos. D adas las múltiples relaciones que pueden originarse en el pensamiento del sujeto que informa o del que se informa, estructuramos la jerarquía de interés a través de lo que se quiere saber de la información, eliminando aquello que por el momento no es útil para significar.

Existe otra forma de significación que podemos llamar asociativa, en donde ningún cambio posible puede influir en el significado de la información que queremos saber. Para todos los fines prácticos, basta creer ciegamente en el por qué y en el cómo del significado que pretendemos perseguir.

Las diversas zonas de significatividad se superponen en busca de una precisión y actúan de forma autónoma, de ahí que posibilitan diversos grados de interpretación. La constante elección y combinación de elementos crea zonas de significación transitoria e inestables, dado que es un proceso de aproximación constante a la significación primaria que se supone conduce a encontrar los conceptos que satisfagan los planteamientos que motivan la búsqueda de la información.

En el proceso de búsqueda se presenta un momento inicial dirigido a elegir los elementos necesarios para crear un grado de significación a partir del cual profundizar 
las indagaciones que servirán de guía. En este primer momento las elecciones establecen el problema o fijan los objetivos respecto de los conceptos o contenidos temáticos que se buscan.

En un segundo momento todos los elementos que definen el problema de búsqueda se distribuyen por medio de zonas de significatividad, dando lugar a un proceso que termina en el momento en que se encuentran los conceptos que satisfacen los significados que se plantearon inicialmente.

En la significación de búsqueda, el "interés a mano" - es decir las intenciones primarias que motivan a un sujeto a realizar un análisis- juega un papel determinante, puesto que como no existe aislado sino que es producto de contextos 0 situaciones sociales, tiene la característica de ser inconstante, de moverse junto con la dinámica del proceso social que lo origina; además tiene un peso diferente para cada sujeto en cada momento, es decir que un individuo puede tener "intereses a mano" dispares, incluso heterogéneos, en cuyo caso el intento por explicar estas características motiva la búsqueda de información.

\section{DE LOS PRINCIPIOS PARA ANALIZAR DOCUMENTOS}

Los modos para representar documentos aluden a cada una de las distintas maneras de darle significado a la representación. El modo más general consistiría en describir un documento con objeto de identificarlo y diferenciarlo de los demás, pues no está de más recordar que la representación de un documento siempre se realiza en función de otras representaciones.

Mencionamos ya que el conocimiento compartido sobre los modos para representar documentos, permite cifrar y descifrar mensajes entre distintos tipos de interlocutores. Estos modos son producto del conocimiento acerca de la naturaleza de distintas clases de documentos, y pueden expresarse en forma de principios generales para describirlas. A partir de ello es factible construir sistemas de reglas o normas cuyos enunciados faciliten la producción e interpretación de representaciones documentales, dado que crean una base común de conocimiento utilizable como punto de referencia para normalizar criterios útiles que sirvan a la descripción de un documento en forma consistente.

Como en la descripción es importante la capacidad de evocación de la palabra convertida en código alfabético para representar algún elemento del documento, resulta entonces que la eficiencia y la economía de códigos se convierten en un factor determinante en la selección de los mismos. De esta manera, el apellido de un autor conformado por unas cuantas letras es eficiente y económico porque puede evocar no sólo a una persona, sino incluso una gama completa de temas -dependiendo de la experiencia del receptor-, aún cuando puede presentarse la ambigüedad en el momento en que un apellido hace referencia a varios sujetos que se desempeñan en distintos campos de la actividad humana. 
La descripción de un documento no debe pretender únicamente enumerar datos, sino buscar convertir sus características en elementos productores de información al servicio de la interrogación del público que utilice la representación. Por tanto, los elementos a incluir en la descripción no se determinan siguiendo la lógica interna de las posibles relaciones entre los datos que la conforman, sino en razón de elementos externos como pueden ser los fines de recuperación establecidos como valiosos, relevantes o pertinentes para que la descripción de un documento cumpla su objetivo.

Por ello, para el estudio de lo que significa describir un documento, no tiene interés el simple abordaje de las formas de aplicación de reglas contenidas en algún sistema - para determinar los elementos y el orden que debe tener una cadena de datos que represente un documento- , porque todo se reduciría a la búsqueda de la solución predeterminada por los enunciados de las reglas. Este tipo de abordaje ha conducido a una concepción técnica de la descripción de documentos que acaba transformándose en un fin que se agota en sí mismo. El estudio de la descripción de documentos debe empezar por cuestionar la naturaleza de los sistemas de reglas, de no ser así ¿cómo indagar el por qué de los mismos?

Cualquier investigación al respecto debe empezar por un por qué y un para qué, cuestionamientos necesariamente referidos a la naturaleza de las finalidades y función que deberá desempeñar la descripción de un documento, así como a las características del mismo. Este tipo de descripción tiene una lógica propia relacionada con el uso y finalidades de la representación documental.

Uno de los objetivos primarios de la descripción radica en poder diferenciar claramente a uno de otros documentos, para lo cual es necesario obtener, como producto del análisis, aquellos elementos que les son propios. Una primera cuestión salta a la vista: ¿cómo iniciar el análisis del documento para determinar sus rasgos particulares?

El significado de la descripción formal es consecuencia de los conocimientos que se tienen acerca de los elementos que se considera contribuyen para lograr que ésta sea relevante. Cuando tales conocimientos se transforman en sistemas de reglas o normas, éstas enuncian lo que se estima significativo para representar un documento. Por ejemplo, considerar que el autor y el editor son elementos significativos para la descripción formal daría como consecuencia la creación de varias descripciones. Los nombres de autores tienen en común su calidad de autores, lo cual crea una relación entre todos ellos, pero además existe un vínculo entre éstos y los editores, así como con la naturaleza del mercado a que se dirigen.

Al delimitar los fenómenos que crean relaciones significativas para un documento, se establece una consistencia que hace factible la relación entre los contenidos de distintos registros. Un registro aislado únicamente permite saber acerca de un documento, de manera que son las relaciones entre los contenidos de varios registros las que dan la posibilidad de establecer un orden documental que hace factible conocer acerca de una colección de documentos. 
Anteriormente indicamos que el análisis para representar un documento a través de símbolos está condicionado por el sujeto que realiza la representación, es decir por las finalidades que persigue, así como por las formas generales de la representación que pertenecen al sujeto que la elabora y no a los elementos del documento. Esto quiere decir que el análisis debe obedecer en una primera instancia a esas formas generales de la representación; por tanto, si para representar documentos es necesario establecer clases para agruparlos, entonces la primera condición del análisis es determinar si existe una clase dentro de la cual sea factible incluir el material que se pretende analizar y en caso de no existir debe empezarse por determinarla.

\section{La inferencia en el análisis documental}

Cuando nos enfrentamos a un tipo de documentos sobre el cual no existen elementos que permitan identificarlo y describirlo es necesario establecer principios generales para su análisis y descripción. A este respecto Ranganathan ${ }^{5}$ menciona que el desarrollo del método para la construcción de principios y de sistemas de reglas que sustenten el análisis y descripción de documentos está condicionado por la presencia de un método científico constitutivo de un ciclo infinito que podríamos caracterizar de la siguiente manera:

1. Aprovechar las experiencias particulares en cuanto a la descripción de documentos como una suerte de conocimiento previo que sirva al propósito de iniciar la construcción de principios generales por medio de la inducción y la aplicación de principios de normatividad documental.

2. Reducir los conocimientos generados a principios normativos, por medio de la intuición y la imaginación.

3. Crear cánones derivados de los principios normativos, por medio de la inferencia y la semántica.

4. Confirmar por medio de la aplicación particular que los principios normativos creados son validos.

5. Volver a iniciar el ciclo.

6. Continuar hasta generar nuevos principios.

Asimismo, Ranganathan señala que la aparición de nuevos tipos de materiales de lectura que aparentemente podrían transcender el ámbito de los principios de análisis documental en uso, deberían abordarse a través de una interpretación apropiada de los principios existentes. En caso de que dicha interpretación resulte inadecuada, sería necesario la construcción de nuevos sistemas de reglas, necesariamente a partir de los principios normativos existentes, a fin de lograr una acumulación de conocimientos.

5 Ranganathan. Classified CatalogreCode India : Sarada Ranganathan Endowment, 1989, pp. 20-21. 
Sería importante revisar los principios del análisis documental en el momento en que se presentara la necesidad de generar servicios - distintos a los existentes en cuanto a organización, finalidades y técnicas-, que no fuese posible construir a partir de los principios existentes. Esta situación haría necesario crear nuevos principios para reemplazar a los anteriores. A este respecto, consideramos que en el caso de la creciente circulación y uso de documentos digitales, nos encontramos frente a una situación que obliga a recrear los principios del análisis documental, pues si bien es cierto que los documentos siguen teniendo esencialmente las mismas finalidades, también lo es que cambian sus medios de producción, consulta y tipos de servicios a prestar.

Un documento digital tiene una naturaleza diferente a los impresos, y su sola utilización por medio de tecnologías distintas genera nuevos conceptos como las relaciones hipertextuales.

Los primeros documentos digitales derivaron de la automatización de los impresos para prestar servicios en línea, y hasta ahora la versión digital ha seguido los mismos principios normativos establecidos para su predecesor, y en algunos casos ni siquiera ésos.

\section{Tipología de documentos y descripción formal}

Las diferentes propuestas para agrupar documentos por clase provienen de los estudios realizados por distintos autores, y se encuentran sistematizadas y vinculadas a la descripción de documentos en los sistemas de reglas y normas; asimismo, los manuales de estilo para edición y publicación establecen su propia tipología que sirve de modelo para la construcción de documentos académicos. Aún cuando existen diversos estudios dedicados a la clasificación documental, es un campo que requiere de profundización, sobre todo respecto a la tipología de documentos aplicada al análisis y representación de los mismos - en áreas temáticas específicas o en cuanto a los nuevos soportes digitales- , pues la transformación que se plantean en cuanto a los criterios de publicación y uso de documentos hacen necesario reflexionar sobre las finalidades del análisis en el contexto de una sociedad informatizada.

La determinación de la clase de un documento implica el conocimiento del significado semántico de las palabras y los términos que se utilizan para aludir a éste; por ejemplo, si el documento en cuestión es un programa de cómputo, el conocer el significado de este término (programa decómputo) ayuda a comprender su naturaleza, ya que para establecer el significado se alude a las características del objeto o concepto, así como a sus relaciones de sinonimia. Esta comprensión previa del documento permite incluso identificar las variaciones que pudieran existir, es decir las probables especies dentro de una clase de documentos.

Los manuales de estilo para editar y publicar constituyen un paradigma acerca de los documentos académicos que resulta de gran utilidad para comprender la 
naturaleza de diversos de tipos de materiales, dado que establecen definiciones que se consideran como modelos a seguir para la construcción de documentos académicos. A este respecto tenemos por ejemplo, el caso del Manual deEstilo de la UniversidaddeChicagoque tiene un carácter general, sin embargo existen manuales de estilo destinados al uso de comunidades académicas específicas.

La tipología de documentos, tanto la expresada por los autores como la contenida en normas y sistemas de reglas, y manuales de estilo, maneja una terminología que pretende estar libre de ambigüedades y facilitar con ello la comprensión. Pero esta terminología no necesariamente corresponde a las formas en que socialmente se utilizan palabras y términos para denominar a distintos tipos de documentos, incluso los nombres que se emplean no son del dominio del lenguaje común. Se trata de un lenguaje especializado útil para comprender y explicar la naturaleza de los documentos, razón por la cual es necesario distinguir entre la terminología propia y la tipología documental; es decir, diferenciar la terminología que es útil para estudiar, comprender y analizar los documentos, y la que se debe usar como parte de la descripción. Así por ejemplo, López Yepez emplea la denominación doamentos sonoros para referirse a los que comúnmente están identificados con el audio (audiocasete). En este caso utilizaremos para describir el documento en particular aquel término que tenga una significación unívoca para el público al cual se dirige la descripción.

Las categorías utilizadas por la tipología documental son de utilidad porque permiten, además, organizar el análisis de un documento. Si se considera, por ejemplo, la clásica división en documentos primarios y secundarios, es fácil percatarse de que a partir de ella se desprenden distintas formas de análisis. En otros términos, aún cuando en esencia las formas de análisis serían semejantes, el uso de cada uno de estos tipos de documentos imprime formas peculiares a la descripción de cada uno de ellos. Mientras que en el caso de los documentos primarios es imprescindible singularizar sus contenidos temáticos para relacionarlos con otros de su misma clase o tema, en el caso de los secundarios resulta deseable describir el alcance de los datos que contiene, así como la confiabilidad de los mismos como instrumento de búsqueda.

Así pues, al analizar un documento para representarlo es necesario, en primera instancia, ubicar algunas categorías y conceptos que no están explícitas en ninguna de sus partes (por lo regular en los libros o programas de cómputo no se dice qué es un libro o un programa de cómputo). No obstante, la naturaleza de un documento se encuentra implícita en él mismo, debido a que su creación, reproducción y uso forma parte de la cultura de una comunidad, cultura que es necesario conocer para poder determinar la clase a la que pertenece. De esta manera, nos encontramos con que las categorías a partir de las cuales se determinan los elementos para representar un documento pertenecen a los modos que tiene una comunidad para crear, reproducir y apropiarse de sus contenidos temáticos. 


\section{SISTEMAS DE REGLAS Y NORMAS}

El propósito de un sistema de reglas o una norma es crear una base común, utilizable como punto de referencia para la normalización de criterios que sirvan a la descripción de un documento en forma consistente y fundamentada en principios generalizados.

La representación documental se sustenta en sistemas de reglas y normas que funcionan como puntos de referencia para su producción e interpretación con la intención no de uniformar las representaciones, sino de imponerles consistencia, es decir hacerlas coherentes estableciendo relaciones entre los diversos elementos que las componen, a fin de que puedan cumplir con las funciones, actividades o fines para las cuales se realizan.

G ran parte de los conocimientos y principios desarrollados en esta materia se han convertido en reglas normativas que tienden a ser codificaciones de las mejores prácticas conocidas. Empero, la influencia de la ciencia, las tecnologías de todo tipo, y el cumplimiento de ideales sociales o modas, han influido en la creación de normas que no necesariamente expresan esas prácticas.

La pretensión de los sistemas de reglas no se reduce a enumerar los requerimientos de la representación, su intencionalidad está dirigida a propiciar la producción de representaciones significativas, productoras de información al servicio de la interrogación del lector. Establecen enunciados lingüísticos que expresan la aplicación de un concepto, o bien proporcionan parámetros para normar la ejecución de un procedimiento. No son ideales a cumplir porque no expresan todas las posibilidades de calidad deseables en la puesta en marcha de una tarea, pues la calidad depende de la competencia y cualidades intelectuales de quien la efectúa.

Los objetivos que se pretende lograr con los sistemas de reglas y normas se centran en la expresión formal de métodos para la representación documental que es necesario que sea, al menos en principio, socialmente deseable.

Un sistema de reglas expresa por medio de formulaciones lingǘsticas el proceso a seguir para cumplir los objetivos que se persiguen con una representación documental. Cada una de estas formulaciones tiene una estructura compuesta de los siguientes elementos:

* Un antecedente en el cual se hace mención del objetivo deseado.

* Un consecuente en donde se señala algo que tiene que o no tiene que hacerse (hay que, debe de).

* Un sistema de relaciones entre las reglas que componen el sistema.

La verificación de las formulaciones contenidas en un sistema de reglas está referida a su carácter empírico funcional, mientras que en el caso de una norma su estructura contempla únicamente un antecedente en el que se expresa un deber ser - para logro de los objetivos deseados- y un consecuente en donde se menciona algo que tiene que 0 no tiene que hacerse. 
Así también las reglas para describir relaciones entre los elementos utilizados para representar un documento. Por ejemplo, la regla que nos dice que "la descripción física de un libro debe contener por lo menos el autor, el título y el año de publicación", nos dice "algo" acerca de lo que significa un libro, a saber: que lo escribe una persona denominada autor, que tiene un nombre denominado título, y una fecha de publicación. También nos dice cómo podemos averiguar acerca de su existencia: simplemente hay que preguntar por su autor o el título. Es fundamental destacar que las reglas casi siempre están demasiado simplificadas, pero que son aceptables como "definiciones nominales". D espués de todo, no puede esperarse que una definición nos diga todo acerca de algo. Por ejemplo, la mayoría de las personas estaría de acuerdo en que un libro implica mucho más de lo expresado por la regla antes citada. No obstante, cuando enfrentamos problemas de representación documental necesitamos apoyarnos en aquellas reglas que nos ayuden a resolverlos.

Siempre debemos tener en consideración el uso de las reglas, a pesar de su simplificación, porque éstas no sólo ayudan al propósito de resolver los problemas de representación documental; su utilización implica, además, lograr una consistencia tal que nos permita relacionar o comparar los elementos vertidos en la representación de documentos. Por ejemplo, si empleamos la regla que dice que para describir un libro debemos referimos al autor, título y fecha, todas las descripciones que se sujeten a ella estarán relacionadas entre sí, en razón de que en todos los casos intervendrán los mismos elementos y relaciones. D e otra forma, si utilizamos una regla para describir el libro 1 y otra para el libro 2, obtendremos dos tipos de descripción que pueden o no relacionarse dependiendo de los elementos y relaciones que intervengan en cada caso.

Es importante señalar que si las reglas aplicadas no relacionan los fenómenos de las descripciones efectuadas, se perdería el sentido de la representación documental: establecer relaciones entre distintos documentos.

La construcción de representaciones documentales tiene un carácter complejo, ya que para expresar las relaciones entre los diversos elementos de la descripción es indispensable recurrir a distintos sistemas de reglas. Así por ejemplo:

* Para la descripción física de los documentos están las reglas para descripción establecidas por la International Standard Book D escription (ISBD's), que es un sistema de uso generalizado.

* Para determinar los asientos para la estructuración de catálogos existen diversos sistemas de reglas, algunos producidos por organismos internacionales de normalización como la ISO y otros de carácter regional como las reglas angloamericanas de catalogación.

- Para describir los contenidos podemos optar entre varios sistemas de reglas orientados a la clasificación de los contenidos: encabezamientos de materia, tesauros, descriptores, resúmenes, y otros. 
Cada una de las normas o sistemas de reglas especifica y delimita su campo de aplicación, con lo cual se establecen criterios que permiten homologary combinar las formulaciones de distintas normas o sistemas de reglas. Las reglas para representar documentos tienen una característica en común: su estructura lógica permite combinar diferentes sistemas de ellas con el propósito de solucionar problemas específicos.

Los sistemas de reglas para representar documentos están constituidos por expresiones lingüísticas a las que se les denomina reglas, cada una de las cuales enuncia los fenómenos referidos a determinado tipo de documentos y los representa mediante téminos que son la expresión lingüística de todo lo que pueda ser objeto de pensamiento o de aquello que pueda darse en cualquier proposición verdadera o falsa.

La representación de la forma y/ o contenidos de un documento nos refiere a sistemas de reglas que determinan los fenómenos y las relaciones que se estiman significativos para la descripción. Sin embargo, la representación únicamente nos permite conocer acerca de un documento, pero las relaciones que puedan establecerse entre la forma y/ o contenidos de varios materiales depende de la organización catalográfica y de la clasificación.

La clasificación delimita un universo documental que permite, en primera instancia, determinar si un documento en particular puede o no incluirse como parte de ese universo, pero además crea un espacio en donde se establecen relaciones lógicas entre los fenómenos representados en los registros que describen a dicho documento; relaciones que dotan de significado a cada uno de esos fenómenos y significados que sirven de base para la organización física de los documentos en sí y sus representaciones. En otras palabras, podríamos decir que la clasificación genera un núcleo de conocimientos, porque al crear relaciones lógicas entre los contenidos de diversos documentos, se obtienen también las relaciones entre los conceptos vertidos en esos contenidos.

El análisis para describir la forma y/ o contenidos temáticos de un documento presenta varias facetas que se realizan simultáneamente, fundamentalmente radica en la habilidad intelectual para reconocer los contextos y significados de un documento, para, en un segundo momento, determinar la aplicabilidad de una regla específica con la intención de obtener una representación simbólica de los contextos y significados del documento en cuestión.

La clasificación, al delimitar un universo documental y crear un espacio donde se establecen relaciones lógicas con la finalidad de crear un orden que haga explícitos los vínculos entre diversos documentos, facilita la organización de éstos y sus contenidos por medio de la formación de colecciones en un lugar específico como puede ser una biblioteca, un centro de información, un archivo, entre otros.

\section{Los principios generales de la nomalización}

Al estudiar los tópicos relacionados con la normalización en el ámbito de servicios bibliotecarios, bibliográficos, de información y redes de información, no ha 
dejado de llamar mi atención el constante esfuerzo de la mayoría de los estudiosos del tema por reducir el fenómeno y aislarlo, en un vano intento por tratar de convencernos de la naturaleza obsesivamente técnica del tema. No obstante, considero que la explicación de los fenómenos involucrados en la realización y uso de normas trasciende los estrechos límites de la apreciación técnica.

La normalización cobra importancia conforme la actividad económica y social se guía más por el cumplimiento de fines y metas que por la referencia a valores; la normalización se convierte así en una necesidad imperiosa destinada a servir de instrumento para establecer un sistema de valoración jerárquica respecto de los fines y metas a cumplir en el ejercicio de una actividad profesional. En la medida en que se abandona el estudio y comprensión de la naturaleza de la representación documental, se hace necesario suplir estos conocimientos con normas y procedimientos técnicos.

El imperativo de la normalización como instrumento para orientar el desarrollo de las actividades profesionales ha conducido a algunos autores a considerarla como objeto de estudio de una disciplina independiente que abarcase los problemas de normalización en todos los ámbitos de la actividad humana, propuestas que han corrido con poca fortuna, puesto que no existen realmente argumentos para sostenerlas. Sin embargo, sí es de llamar la atención el aumento de la actividad normativa que obliga a diversas disciplinas a desarrollar un campo de estudio dedicado a las normas y a la actividad de normalización.

En este campo de estudio se emplean palabras como norma, reglas, sistema de reglas, normalizar, homologar, normativo, normalización, competencia, actividad, procedimiento, técnica, tecnología, uniformidad, consistencia, transferir, protocolos, normar, formatos, estándar, standard, standardizar, standardización, para referirse a objetos, conceptos y métodos inherentes a este campo. Por ello, al utilizar estas palabras desde la perspectiva de la normalización les atribuiremos un significado unívoco con el fin de establecer una relación de significado entre un objeto 0 un concepto y la palabra en cuestión, independientemente del que puedan tener en términos semánticos o en cualquier otra disciplina o campo de estudio.

\section{¿Qué es normalizar?}

En un sentido general normalizar, según los diccionarios de la lengua, significa regularizar o poner en buen orden aquello que no lo está. Si normalizar implica poner en buen orden, entonces, cuando nos referimos a normalizar en el ámbito de estudio de la representación documental, estamos implicando la idea de ordenar lo desordenado. Cabría preguntarse si este significado semántico de normalización coincide con los fenómenos propios de normalizar en el campo de la representación documental. En términos generales podríamos responder afirmativamente, aún cuando habría que hacer algunas precisiones.

Normalizar alude fundamentalmente a la pretensión de lograr productos y servicios equiparables, es decir que sea factible distinguir semejanzas y diferencias con 
otros productos y servicios. Para que éstos puedan compararse fácilmente se recurre a productos o servicios tipificados y ordenados por clase en torno a elementos comunes claramente identificados. En este sentido, normalizar implica la acción de organizar conforme a determinada tipología que ha sido aceptada como norma para simplificar y obtener una mayor eficiencia en el rendimiento del producto 0 en los resultados del servicio.

Este concepto implica varios elementos, tales como la aceptación de normas para simplificar el diseño, la construcción y uso de productos y servicios, así como la presencia de instituciones o círculos sociales encargados de producir y validar normas - conforme a metas y fines que se establecen como comunes y social o comercialmente deseables- .

En otros términos, normalizar incluye un conjunto de acciones orientadas a organizar productos y servicios conforme a metas y fines comunes, establecidos en una norma avalada por una institución o un círculo social, con objeto de lograr una mayor eficiencia en el uso de productos y servicios.

Normalizar tiene que ver con el establecimiento de enunciados cuyo propósito es crear referentes que sirvan como guía para el desarrollo de actividades profesionales específicas, con la finalidad de hacer funcional un producto o un servicio; implica una conciencia de que la actividad profesional que se realiza no sólo incumbe a quien la efectúa, y por tanto debe estar referida a fines y metas de fácil compresión y acceso a todos los involucrados en la actividad profesional en cuestión. La belleza de los estándares radica en su volumen, es decir en la posibilidad de contar con una amplia gama para escoger.

El poder seleccionar estándares parecería un contrasentido, sin embargo no lo es ya que implica enfrentar el problema de la normalización como un proceso dinámico y dialéctico, dado que se necesita de estabilidad para poder desarrollar procesos tecnológicos. Ello no obstante, la estabilidad tendería a propiciar el estancamiento del conocimiento y los procesos tecnológicos, de ahí que la inestabilidad sea necesaria para el desarrollo. Por ello los procesos de normalización implican procesos inestables, que a lo sumo pueden aspirar a establecer criterios para facilitar la homologación de procesos tecnológicos.

El campo de la normalización comprende el estudio de las acciones necesarias para aplicar normas y para generar políticas y procedimientos que lleven a la obtención de productos o servicios normalizados a través de métodos apropiados. Evidentemente, también es materia de estudio los métodos y procedimientos para construir y validar normas, así como el abordaje de las normas en sí mismas.

Como la idea de normalización surgió y se desarrolló en el mundo anglosajón, los términos para designarla se tomaron del inglés, se castellanizaron y dieron lugar a las siguientes denominaciones:

standard que se le atribuye el significado de tipo o modelo, es sinónimo de noma 
standardizar, que significa producir conforme a normas, es sinónimo de normalizar, y

* etandardizacoóncuyo significado es estandardizar, es sinónimo de namalización

Preferentemente utilizaremos los términos norma, normalizar, y normalización, porque su fonética es más bella, aunque existen autores que se inclinan por utilizar standard para diferenciar el campo de estudio y referirlo exclusivamente a lo tecnológico, dado que el uso del vocablo norma y normalizar tiene otros contenidos semánticos en Filosofía y en D erecho. Situación sobre la que sí vale la pena abundar.

\section{Principios de normalización}

Los principios generales de normalización deben considerarse al margen de cualquier finalidad tecnológica y atender sobre todo al cumplimiento de finalidades socialmente deseables en el sentido de lograr productos útiles para el bienestar de la comunidad.

Entendemos por productos útiles aquellos que sean fáciles de comprender, aprender, usar y aplicar a la solución de problemas o a la satisfacción de necesidades. Para ello es necesario construir sistemas documentales conforme a los siguientes principios:

\section{Consistencia}

* Que en los sistemas documentales una misma cosa se haga de la misma manera.

* En el uso de signos y símbolos es necesario atender a los significados culturales.

* No importa la manera en que un sistema documental se encuentre técnicamente organizado, sino que esté construido de tal manera que su contenido y operación sea fácilmente predecible para quien intente utilizarlo.

\section{Retroalimentacion}

* Debe contar con elementos de guía y ayuda para el usuario (todo tipo de manuales, ayudas, tutores, preguntas frecuentes, etc.).

\section{Estructura}

* La estructura del sistema debe de ser consistente en el sentido de la descripción y asignación temática, aun en los errores. D ebe tener una estructura lógica fácilmente comprensible en su totalidad.

* D ebe hacer explícitas las finalidades que trata de cumplir y las funciones por medio de la cuales es factible hacer operativo su cumplimiento,

* Debe ser eficiente para los propósitos que fue hecho. 
A continuación se describen algunas técnicas que ayudarían a configurar un buen sistema normativo para el análisis documental.

Los sistemas deben construirse teniendo en mente la homologación a otros sistemas de manera que ésta se convierta en una fácil y rápida transformación de elementos, incluso es necesario señalar aquéllos que no tienen equivalencia en otros sistemas, también si el sistema, por sus características, no es homologable o sólo lo es parcialmente. En cualquiera de los casos se requiere señalar las formas para homologar los datos y procesos.

No podemos ignorar desde luego la importancia que reviste para algunos el que los sistemas sean diferentes. En ello se encuentran envueltas estrategias de mercado orientadas al logro de mayores beneficios, 0 bien intereses institucionales que apuntan a la intención de mantener una determinada posición considerada estratégica.

Sin embargo, inevitablemente, los días del oscurantismo en cuanto al proceso de datos bibliográficos está llegando a su fin y a los usuarios realmente les tiene sin cuidado el porque los sistemas tienen que ser diferentes; no les interesan ni las estrategias de mercado, ni las posiciones de las instituciones, y han empezado a generar sus propios sistemas, situación que debería ser motivo de reflexión tanto para los bibliotecólogos, en su carácter de profesionales de estos asuntos, como para las bibliotecas -instituciones en pleno acomodo de su funcionalidad social- .

Existen sistemas de reglas con una larga y venerable tradición que se utilizan como medio para formalizar y expresar las condiciones indispensables para construir un procedimiento que satisfaga el cumplimiento de un fin deseado (clasificar, describir un documento, elaborar un catálogo, etc.). Difieren esencialmente de las normas en tanto no son patrones o modelos a seguir, ni establecen metas o fines. Los sistemas de reglas son enunciados procedimentales, no describen, ni explican, ni predicen ningún hecho, sencillamente tienen el propósito de orientar la ejecución de determinado tipo de procedimiento y especifican las condiciones para que ésta pueda tener lugar.

La palabra regla tiene varias acepciones: instrumento para trazar líneas, precepto o prescripción, instrumento para medir, etc.; también significa regular, poner en orden una cosa. Se habla de reglas para referirse a los principios que rigen la enseñanza de un arte o ciencia, acepciones estrechamente vinculadas al sentido que se le da en la práctica bibliotecaria, no obstante, aunque el sentido semántico de la palabra regla nos acerca al concepto de instrumento de regulación para obtener un determinado orden documental, consideramos que sólo una aproximación a la naturaleza lógica de los sistemas de reglas puede servirnos para comprender y explicar su razón de ser.

$\mathrm{Si}$ consideramos las reglas independientemente de factores como la forma que adoptan, el sujeto creador o el destinatario, encontramos que una regla sólo existe como tal desde el momento en que adquiere un carácter verbal. Esto quiere decir que la regla lo es en tanto es susceptible de expresarse. La regla es el significado de 
una expresión lingüística. Es una proposición. No toda proposición es una regla. Para tener el carácter de regla es indispensable que se inseteenunsistema proposicianal expresivo de un ámbito en el cual (tiene que) tener lugar determinado tipo de procedimientos.

Una regla considerada en forma aislada tiene una significación ambigua o carece de significado, por ejemplo la regla que asigna la notación 200 para el tema religión, únicamente es comprensible y explicable en razón del sistema de clasificación D ecimal de Melvil Dewey. Las reglas que componen un sistema no pueden desigarse de él, ya que sólo tienen significado en cuanto se entrelazan para formar el sistema. Viceversa: el sistema no puede ser pensado sin las reglas, puesto que el sistema no es sino un conjunto de expresiones lingüísticas dirigidas, directa 0 indirectamente, a orientar la creación de procedimientos específicos.

Todas las reglas de un sistema son proposiciones constitutivas del mismo, y por tanto son proposiciones necesarias, o reglas que establecen un tener que, cuyo contenido se orienta a la instauración de procedimientos que es necesario realizar si se desea cumplir determinado fin. Para poder describir un procedimiento tipificado por las reglas de un sistema, habremos de valernos forzosamente de las mismas reglas que lo constituyen, sistematizando e interpretando el contenido de éstas. De la misma manera, la definición de un sistema en particular, o bien una clase de sistemas, la podemos efectuar mediante el ordenamiento e interpretación exhaustiva del contenido de sus reglas.

Las reglas para representar contenidos temáticos y descripciones de documentos no describen ni explican. El contenido de las reglas, aquello que debe o puede o tiene que hacerse 0 no hacerse, indica el campo de aplicación y las condiciones que tienen que darse, de tal modo que la acción de representar documentos pueda ser calificada como tal. Estos contenidos tienen un carácter newarioya que la finalidad deseada -representar un documento- sólo es posible si se cumplen las reglas del sistema escogido para ese propósito.

Un procedimientoimplica una forma de acción, incluye una serie de elecciones y presupone un campo de aplicación (la biblioteca, una red de información) el cual puede estar sumamente formalizado mediante el establecimiento de límites estrictos o de caracteres fijos e invariables (como en Las Reglas Angloamericanas); 0 puede ser, por el contrario, vago en su delimitación, como en el caso de los tesaurus. Los procedimientos que pueden desprenderse de un sistema de reglas dan por sentado que los sujetos que habrán de constituirlo y ejecutarlo existen y que son competentes para hacerlo; tienen además un carácter dinámico que hace posible su identificación con una acción y sus resultados.

El que el procedimiento pueda elucidarse con la acción y sus resultados da lugar, en ocasiones, a conclusiones erróneas acerca de la naturaleza de los sistemas de reglas. Normalmente la ejecución de un procedimiento produce un resultado, es por ello que cuando expresamos: “alguien efectuó un procedimiento para clasificar 
documentos, conforme a las reglas de un sistema", suele traducirse por: "alguien ha conseguido clasificar estos documentos". Si pensamos en términos ideales en la acción de clasificar -como realización de un acto o conjunto de actos considerados unitariamente- , lo que resulte de la ejecución de estos actos (la organización documental) no es lo mismo que su consecuencia (la posibilidad de buscar determinados documentos, o su posible contenido, y conocer su ubicación física). El resultado es un componente intrínseco del acto o actos que conforman el procedimiento para clasificar, mientras que la consecuencia es el efecto del resultado $y$, por ello, no constituye un elemento del acto o actos realizados sino que se sitúan fuera de ellos y en relación directa con las finalidades perseguidas.

Una definición parcial de noma podría ser que es aquella que tiene como resultado que algo deba o pueda o tenga que ser o no ser hecho. Las formulaciones de normas, en un sentido lingüístico, son un grupo muy variado; utilizan varios tipos gramaticales de sentencias, sin agotar ni ser exclusivamente agotados por ninguno en particular. D ebemos, por tanto, estar prevenidos frente a la idea de basar el análisis conceptual de las normas en un estudio lógico de determinadas formas lingüísticas de discurso.

Q ue una sentencia sea o no la formulación de una norma jamás podría decidirse sobre fundamentos "mórficos", es decir, sólo con base en el signo. Esto sería así, sólo si existiera una clase precisa y delimitada gramaticalmente (morfológica o sintácticamente) con expresiones lingüísticas cuya función "normal" o "propia" fuera la de enunciar normas. Pues aun en este caso sería el usode la expresión y no su aspetolo que determinaría si es la formulación de una norma u otra cosa.

Cuando decimos que es el uso y no el aspecto de la expresión lo que muestra si es la formulación de una norma, estaríamos de hecho diciendo que la noción de norma es previa a la noción de la formulación.

Es un requisito lógico de las normas el que sea posible que las personas puedan cumplir las exigencias que éstas le imponen. A unque de mayor interés para la discusión de las normas, en el presente trabajo, es la aplicación, a las normas técnicas, del principio: db̋, entraña, puede

Las normas técnicas en términos aproximados guardan relación con los medios a emplear para alcanzar un determinado fin. Las reglas contenidas en ellas presuponen que las personas que las siguen aspiran a un fin o resultado. Veamos.

La formulación tipo de las normas técnicas encierra oraciones condicionales, en cuyo antecedente se señala alguna cosa que se desea, y en las que en su consecuente se menciona algo que tiene que (hay que, debe de) o no tiene que hacerse.

Supongamos que la norma sea:

si quiero conseguir un determinado fin $\underline{\mathbf{E}}$

tengo que hacer un determinado acto $\underline{\mathbf{A}}$

Como existe la posibilidad de que quiera conseguir este fin independientemente de que pueda o no hacer cualquier acto necesario para su logro, sucede 
entonces que $\underline{\mathbf{E}}$ es algo que deseo, pero que puede significar varias cosas.

Puede significar por ejemplo:

Q ue E es algo que recibiría "con agrado" si me sucediera, ya sea como favor del destino 0 gracias a la acción de algún otro agente. En este sentido $\underline{\mathbf{E}}$ puede ser una cosa que yo deseo, aún cuando no pueda hacer lo necesario para su obtención.

Q ue $\underline{\mathbf{E}}$ es algo que deseo, puede significar que ansío que $\mathbf{E}$ me suceda. Esto puedo tenerlo sin ser capaz de poner los medios para obtener $\underline{\mathbf{E}}$.

Pero desear algo puede también significar perseguirlo como fin de la acción.

Una inferencia práctica se da cuando una persona extrae de una norma técnica una prescripción para su propia conducta.

A las directrices las llamamos también normas técnicas, dado que presuponen fines de la acción humana y relaciones necesarias de los actos con estos fines.

Una norma representa requerimientos mínimos aceptables en la realización técnica de un trabajo, no representa un ideal a cumplir porque no expresa todas las posibilidades de calidad o deseables en la ejecución de una tarea, muchas de las cuales dependen de la calificación profesional de quien realiza el trabajo y de su habilidad para ligar los procedimientos técnicos con los requerimientos.

La idea de una norma es la de imponer consistencia y no uniformidad, entendiendo por consistencia que existe cohesión, relación entre varias cosas. Las normas se construyen y justifican en razón del cumplimiento de una función determinada. ¿Funciona o no? es constantemente la pregunta. Una de las consideraciones a tomar en cuenta es que el deber ser de una norma técnica, en cuanto a los objetivos que se pretende lograr con su aplicación, tiene que ver con ser necesaria (al menos en principio socialmente deseable) para que pueda cumplir sus funciones sociales previstas. De otra manera caería en el vacío social.

\section{Normalización}

La normatividad tendría como finalidad crear normas para ayudar a regular la aplicación, transformación, el desarrollo y la adaptación de la tecnología, y no para establecer un control del desarrollo tecnológico, ya que un acto así tendría por consecuencia eliminar la creatividad en la aplicación de la tecnología y dificultaría su transformación.

Las normas abordan universos limitados y tienen reglas explícitas para relacionar los procedimientos tecnológicos empleados en la solución de un problema 0 en la realización de una actividad. Tienen como finalidad solucionar los posibles conflictos que surgen al aplicar procedimientos distintos a los habituales.

La mayor parte de los problemas de normalización no son en sí mismos conflictos técnicos, sino que derivan de la forma en como las personas aplican los procedimientos técnicos para resolver determinadas necesidades en torno a la creación de acervos y medios para manejarlos. 
La normatividad estaría orientada a la formación de conceptos a partir de los cuales puedan derivarse actividades formales de carácter unificador de técnicas (normas) y conceptos (finalidades), así como al fomento de las actividades de normalización informal (docencia, investigación, terminología, acuerdos institucionales).

Las normas son instrumentos esenciales para la normalización, pero como representan respuestas concretas a problemas específicos, es menester establecer generalizaciones que definan los principios con base en los cuales se habrían de articular diversas técnicas derivadas de distintas normas, y poder así formular un todo coherente. Las normas deben cumplir la misma función de puntos de referencia que cumplen los diccionarios de una lengua.

Los componentes de la normatividad deben sustentarse en la construcción de principios generales cuyo objetivo primordial sería hacer hincapié en la acción unificadora de conceptos por encima de cualquier noción tecnológica específica. No sería ajena tampoco al enunciado de juicios cualitativos que sirvan para evaluar la forma y objetivos de un sistema de información documental, y abarcar los diferentes aspectos de la amplia gama de fines e intereses a cubrir por una organización, porque la normalización únicamente adquiere sentido cuando se le vincula al uso y destino de los documentos.

Aunque estamos acostumbrados a evaluar sobre la base de elementos cuantitativos, es indispensable encontrar los medios cualitativos adecuados para que en conjunto nos ayuden a determinar si un sistema de información es el indicado para disponer de la información documental.

La configuración de conceptos que sirvan como medios para decidir acerca del uso de determinadas normas, no se reduce a un problema de enumeración de criterios de selección en torno a la aplicabilidad monolítica de una norma en detrimento de otra, pues abarca una gama más amplia de conflictos que tienen que ver con la definición de criterios y lineamientos para el uso de técnicas y procedimientos de almacenamiento y recuperación.

La creación de conceptos normativos dirigidos a obtener la eficacia, así como la creación de normas técnicas, apoyan fundamentalmente el desarrollo de la industria de la información. Es el caso por ejemplo del International Standardized Book Number (ISBN) que crea un número normalizado para facilitar la identificación de los títulos publicados; o el International Standardized Serials Number (ISSN) que otorga un número normalizado a cada una de las revistas que se publican; o el caso del CODEN, un número normalizado que facilita la codificación de publicaciones seriadas.

Todas las normas técnicas relacionadas con los números normalizados están dirigidas a facilitar la codificación de datos para usos automatizados; sus objetivos son estrechos y quedan sujetos a las posibilidades del desarrollo tecnológico en este terreno. Un caso típico a este respecto es la norma ISO-2709 que establece un procedimiento para realizar formatos de captura de datos bibliográficos; a partir 
de ella se han realizado los formatos MARC y CCF, pero ambos han sido superados por las posibilidades técnicas actuales, y como son procedimientos ligados a determinada tecnología, su modificación resulta complicada, por lo que hoy crean más problemas que soluciones.

El incremento en las cantidades de información disponible trajo consigo la idea de que el volumen, en mayor o menor medida, es un factor importante en la transmisión de información. Como consecuencia, en el campo del control bibliográfico o documental se llegó a estimar que necesariamente la información reduce la incertidumbre. Esta manera de pensar se ve reforzada sobre todo porque los sistemas de control hacen demasiado énfasis en la organización de la información y su provisión al usuario, pero excluyen cualquier consideración respecto a los fenómenos de comunicación presentes en el proceso de análisis y representación de documentos.

Prestar una mayor atención a los hechos que gobieman la producción, provisión y recepción de información documental, considerándolos como parte de un proceso de comunicación orientado a satisfacer la necesidad de conocer determinados documentos, nos permite situar el análisis y representación de documentos en el centro de un ambiente informativo determinado; $y$, con ello, aumentar las posibilidades de que sea útil en la familiarización con distintos campos del conocimiento - campos relacionados con el cumplimiento de objetivos institucionales o de los posibles usuarios de un acervo documental-.

En este sentido, la normatividad de clasificación y descripción documental debe proveer instrumentos para determinar y evaluar la eficiencia técnica de un sistema de control bibliográfico o documental en razón de las respuestas que pueda proporcionar al público, y dirigir la evaluación hacia efectos favorables (rentabilidad, productividad, etc.), pero más en razón de las pérdidas que evitan, que de las ganancias o provecho obtenido. Por ejemplo, si un pasajero tiene una tabla de horarios y rutas de trenes y aviones, eso no hará que el sistema de transporte vaya más rápido, pero puede evitar esperas innecesarias. La información sobre rutas y tiempos puede ayudar a facilitar el viaje.

Los servicios de información documental, medidos por el grado de prevención de pérdidas, ayudan a configurar un esquema normativo del control bibliográfico o documental cuya intención principal sea prevenir pérdidas innecesarias.

Medir los efectos de un sistema de información documental por medio de las pérdidas que evita, cuando la comunidad a la que sirve está bien informada, y no sólo con base en la cantidad de información que es capaz de captar y organizar, significa crear esquemas normativos de evaluación cualitativa.

Generalmente los sistemas de información documental responden a criterios normativos cuantitativos con los que se evalúan sus actividades en función del número de servicios prestados. Ahora bien, si la normatividad pretende incluir medios cuantitativos y cualitativos, sería necesario definir los procesos en torno a 
los cuales se habrá de fundamentar la percepción de la información. Tenerlo claro es indispensable para codificar y representar - tanto la descripción del documento como sus contenidos temáticos- , así como para derivar aquellos elementos cualitativos que habrán de incluirse en el deber ser de la normatividad durante el proceso de análisis y representación de documentos.

\section{Nomalización y desarrollo tecnológico}

A pesar de los indiscutibles avances que constituyen las normas como instrumentos para representar documentos, únicamente ahondan en un problema ya conocido, sin proporcionar posibles soluciones a cuestiones derivadas del momento en el cual el público tiene necesidad de definir los elementos necesarios para orientar el rastreo de información.

La normatividad en el ámbito de la representación documental implica la definición de un dbəer serineludiblemente vinculado a la determinación de objetivos a perseguir. Su intención debe encaminarse a convertir la representación en un medio para facilitar la distribución social del conocimiento y lograr que el público esté bien informado.

En última instancia, la normatividad para representar documentos forma parte del diberseracerca de las finalidades y funciones de la preservación y organización del conocimiento registrado por medio de todo tipo de documentos, es decir, fundamenta su actuación en fines y valores socialmente aceptados; de estos últimos se derivan los elementos constitutivos de principios encaminados a establecer una normatividad cuya intención sea servir de guía para instrumentar una organización documental funcionalmente dirigida a cumplimentar los fines y valores de la preservación y difusión del conocimiento.

Por esta razón, la definición de elementos teóricos en tomo a la representación documental debe concebirse como una función evolutiva que permita englobar en un todo coherente los elementos relevantes para el cumplimiento de los fines asignados a la representación de un documento, sin perder de vista su destinatario final, porque es el público el que en ciertas condiciones y en algún momento le habrá de dar significado a la funcionalidad, formas y modos de la representación documental.

Cuando se piensa en la representación documental únicamente como un medio para describir, y clasificar y formar colecciones de documentos como parte de un simple formalismo, aislado o al margen de cualquier consideración sobre la estructura que se está creando, sin ocuparse de las condiciones sociales que facilitan 0 impiden su utilización, se está contribuyendo a formalizar estructuras documentales carentes de sentido, porque las colecciones, por bien estructuradas que estén, por sí mismas, aisladas de su contexto social, no tienen significación de ningún tipo.

La creación de nuevas opciones para representar documentos es un imperativo derivado de la dificultad de satisfacer las demandas de información generadas por 
el público, porque en los hechos la representación se ha desplazado hacia la captación y representación del contenido del documento, con base en su representación por medio de códigos descriptivos.

Como indica Schutz, "la principal característica de la vida de un hombre en el mundo moderno es su convicción de que, en conjunto, su mundo vital no es totalmente comprensible para él ni para ninguno de sus semejantes. Existe un acervo de conocimiento teóricamente disponible para todos, acumulado por la experiencia práctica, la ciencia y la tecnología como concepciones fundamentales. Pero este acervo de conocimiento no está integrado; consiste en una mera yuxtaposición de sistemas de conocimiento más o menos coherentes, que por su parte no son coherentes, ni siquiera compatibles unos con otros." 